\title{
ESCALERA REYES, JAVIER y COCA PÉREZ, AGUSTÍN (Coords.) (2013). Movimientos sociales, participación y ciudadanía en Andalucía. Sevilla: Aconcagua Libros, 308 pp.
}

\author{
Félix Talego Vázquez \\ Universidad de Sevilla
}

En el número 2 de esta misma revista hemos tenido ocasión de comentar un libro editado por Aconcagua, "Consumir naturaleza. Productos turísticos y espacios protegidos en Andalucía". Hoy comentamos otro de la misma editorial: "Movimientos sociales, participación y ciudadanía en Andalucía”, que reúne textos de divers@s investigadores sobre experiencias, perfiles, potencialidades y limitaciones de la participación ciudadana en procesos de decisión pública. Ambos libros están centrados en Andalucía y, aunque con temática distinta, los dos suponen un fresco de la sociedad civil de nuestro tiempo, complementarios pues.

En algunos de los casos que se presentan, los propios investigadores se han implicado en los procesos de participación de los que dan cuenta, trascendiendo el rol "investigador" para "actuar" y "participar" sobre la realidad social estudiada: Investigación-Acción Participativa (IAP), que así se llama la metodología, cuya concepción y fases se explican en el primer capítulo: “Teoría y práctica de la participación”. Lo escriben Javier Escalera y Agustín Coca, a su vez los coordinadores del libro.

La propuesta que anima los diferentes capítulos y que da unidad al conjunto es que la participación es un requisito imprescindible de cualquier proceso de democratización; que esa participación, para ser efectiva, ha de estar organizada con una metodología que no la deje a la espontaneidad y al albur de las circunstancias, y que, precisamente 
la IAP ofrece un marco adecuado con el que poder desarrollar propuestas adaptadas a diferentes contextos de participación ciudadana. Constatan los coordinadores la escasa "cultura de participación" de la ciudadanía andaluza y española en general, apuntando algunas de las causas del déficit: no solo la obstrucción legal y el desierto participativo del pasado franquista (a excepción de las organizaciones clandestinas, mal toleradas los años finales de aquel régimen), sino el escamoteo de esa participación por los partidos de gobierno en el actual régimen democrático (que nosotros consideramos más bien una partitocracia). Según Escalera y Coca, no ha habido ni hay una educación que anime y forme en participación, lo que juzgan imprescindible para que puedan florecer experiencias de este tipo. Y junto a la educación, una noción de comunidad o identificación compartida, condición esta tampoco fácil, pues la socialización y las dinámicas conducen a una creciente individuación. Frente a la insuficiencia participativa y a las tendencias individualizadoras, la IAP ofrece, según los coordinadores, “....un marco para la producción de procesos deliberativos de construcción colectiva de conocimiento y acción orientados a la transformación democrática..." (p. 23). En el resto del capítulo se ofrecen, con criterio pedagógico, algunos de los requisitos y claves que deben estar presentes en los procesos de participación que quieran implicar realmente a diversos actores y a la ciudadanía en general.

El segundo capítulo lo escriben también Agustín Coca y Javier Escalera: "Espacios naturales protegidos, planes de desarrollo sostenible y participación en Andalucía”. En la introducción al mismo resumen el modelo típico seguido en las políticas públicas de protección ambiental en el Estado español, que solían desconsiderar y desde luego excluir del proceso decisorio a las poblaciones locales, según un esquema que recuerda el modelo de los espacios protegidos del ámbito colonial anglosajón: concibiendo los espacios a proteger como naturaleza agreste no humanizada y en la que "los naturales del lugar" eran poco más que un obstáculo o una amenaza al supuesto "equilibrio natural". Es el mismo modelo que sirvió para regular los primeros parques en territorio de los EEUU (Guha 2000) y que ha servido de referente para tantos casos europeos, el español entre ellos. Los responsables políticos no han considerado la posibilidad de ofrecer espacios de participación a las poblaciones concernidas hasta que no se impone en el lenguaje y las prácticas de las instancias internacionales la noción de "desarrollo sostenible", pues este necesita, teóricamente al menos, incorporar a las poblaciones locales para regular el territorio. En el desarrollo del capítulo se hace un balance de estas iniciativas en Andalucía, con especial detenimiento en tres casos: Sierra Mágina, Parque Natural de los Alcornocales y Parque Nacional de Doñana. Para cada uno de los casos se describe el proceso, el grado efectivo de implicación de distintos sectores poblacionales y los obstáculos institucionales y políticos que han limitado la potencialidad de las metodologías de IAP implementadas. Es sintomático que, en los tres casos tratados, la participación ciudadana desbordara las previsiones y que, al final, los responsables 
gubernativos frenaran o incluso puentearan las propuestas que iban madurándose en los foros.

El tercer capítulo, escrito por Beltrán Roca y Luis Baños, está dedicado a la participación obrera en el ámbito de las relaciones laborales y lleva a cabo una panorámica sobre la historia reciente y los precedentes franquistas del mapa sindical en Andalucía. Es necesariamente un recorrido breve y sinóptico, pero está bien trabado y logra subrayar los hitos fundamentales y los actores más relevantes y más significados según los diferentes conceptos de sindicalismo que han venido repartiéndose el espacio de las relaciones laborales en Andalucía. Articulando los conceptos de "identidad colectiva" y "cultura del trabajo" aventuran una definición de "cultura sindical": "conjunto de percepciones, actitudes, significados y prácticas sociales compartidos por sectores de la población trabajadora en relación al papel de las organizaciones sindicales en las relaciones laborales... los significados compartidos y los recursos colectivos utilizados para pensar sobre el sindicalismo y el mundo del trabajo" (p. 87). En la segunda parte del texto de Roca y Baños encontramos un análisis más minucioso de dos ejemplos de sindicalismo minoritario que subsisten en Andalucía: el sindicalismo jornalero articulado en el Sindicato de Obreros del Campo (SOC) y la cultura sindical del sector industrial de astilleros en la Bahía de Cádiz, con presencia significada de la Confederación Nacional del Trabajo (CNT). Se trata en ambos casos de un sindicalismo más combativo, con expectativas más ambiciosas de transformación social, con formas de participación asamblearias articuladas a un liderazgo fuertemente personalista, en especial en el caso del SOC, hoy llamado SAT (Sindicato Andaluz de Trabajadores). Los autores definen este sindicalismo como "sindicalismo de movimiento social" y muestran finalmente como las movilizaciones del 15-M han supuesto un revulsivo y reactualización de su potencial.

Assumpta Sabuco escribe sobre feminismo, o más concretamente sobre asociacionismo de mujeres en Andalucía, lo que no es exactamente lo mismo, como la autora precisa. $\mathrm{Su}$ texto, titulado "El papel de las asociaciones andaluzas de mujeres y el feminismo transnacional: a modo de reflexión", aborda sumariamente cuestiones de carácter general, como las bases culturales y dialógicas ("construidas"), que no biológicas ("naturales"), de las diferencias en las relaciones sociales de los sexos. Pero, paulatinamente, la autora va centrando al lector en las características, tipos y problemática del asociacionismo de mujeres en Andalucía y su engarce en los esquemas, temas y debates del feminismo trasnacional. Hay en el texto diversos abordajes de las asociaciones incorporando una cierta perspectiva temporal, lo que nos permite hacernos una idea del mapa asociativo de mujeres en Andalucía. Es subrayable por ejemplo su incisivo análisis del rol desempeñado por el Instituto Andaluz de la Mujer; o las dificultades que estas entidades femeninas han tenido y tienen en la participación en entidades gubernativas, donde demuestra un conocimiento sólido, como corresponde a una investigadora que ha tenido incluso 
responsabilidades de asesoramiento político en la materia.

Con Ángel del Río somos llevados a una panorámica privilegiada del movimiento por la recuperación de la memoria histórica en Andalucía. Su texto aúna, con solidez contundente, lo analítico y lo descriptivo, las claves locales y el engarce del movimiento en la problemática memorialista general y aun en el más amplio espectro de los nuevos movimientos sociales. Tal perspectiva privilegiada solo puede alcanzarse tras muchos años de investigación en el tema. Sabemos de su dilatada trayectoria investigadora, que atestiguan abundantes publicaciones y documentales sobre el memorialismo, que son referente para antropólog@s e historiador@s que trabajan en ello; y sabemos también que su compromiso investigador se inserta en un compromiso de fondo, ético y político, contra la impunidad y por la restitución de la dignidad a las víctimas de crímenes de lesa humanidad. Se aúnan en Ángel la condición de investigador y activista, y una fertiliza a la otra, justo en el sentido que defienden los coordinadores en el primer capítulo. En fin, es difícil referir aquí la amplitud de temas y enfoques que se articulan en lograda síntesis en este capítulo, titulado "Nuevos sentidos del pasado franquista. Las políticas de la memoria en Andalucía": lo que significa el concepto de "víctima" como nuevo actor y sus potentes repercusiones en orden a la legitimidad del régimen democrático surgido de la transición; el memoricidio segundo que ha supuesto la transición al régimen democrático actual; la propia noción de "memoria histórica" en comparación con la de "memoria clandestina"; la génesis y desarrollo de las asociaciones memorialistas y sus bases sociales como un caso de asociacionismo civil autónomo; las relaciones de estas con las instituciones y los partidos políticos; el entramado ritual que ha emergido en el memorialismo andaluz, la resignificación de los "lugares de la memoria" y su virtualidad evocadora, provocadora e invocadora.

El Grupo de Estudios Antropológicos La Corrala escribe el capítulo titulado "Ciudad capitalista y conflicto. Movimientos sociales urbanos en Andalucía”. Se trata de un interesante análisis de las iniciativas ciudadanas más o menos organizadas que pretenden contestar a la drástica remodelación de las ciudades andaluzas que han venido consintiendo los poderes públicos ante el empuje de los grandes grupos inmobiliarios y de distribución. Es un texto fresco, que aporta abundantes referentes de movimientos y de acciones, con especial detenimiento en las actividades y significación de las casas y centros sociales ocupados, o la lucha contra los desahucios. Demuestran los autores rigor y profundidad en el análisis, pero, a la vez, compromiso: las informaciones que traen y el ángulo de su tratamiento evidencian que ellos están implicados en este tipo de acciones. Tenemos, pues, también en este caso, esa simbiosis fructífera por la que abogan los coordinadores: investigación-acción participativa. Aunque el capítulo tiene muchas vertientes bien integradas por los autores, queremos destacar aquí una de ellas: la que nos muestra el modo "natural" en que han llegado a hibridarse en la protesta y en las 
propuestas alternativas de nuestras ciudades la vindicación de los derechos civiles y de las personas, lo ecológico, lo feminista, lo pacifista, uniéndose todo ello en los perfiles de 1@s activistas y de sus organizaciones. Y ello sin anular el vector obrero tradicional, pero integrándolo en un conjunto que ya no puede ser llamado movimiento obrero.

Prosigue el libro con un capítulo sobre la participación de la ciudadanía en la conformación y gestión del hábitat urbano. Lo firman Glenda Dimuro, Marta Soler y Esteban de Manuel. Es destacable en este texto la bien lograda articulación de teorías sobre el hecho urbano, la conformación del urbanismo contemporáneo y los planteamientos básicos de la agroecología, con un seguimiento etnográfico solvente de las experiencias de agricultura periurbana ("huertos urbanos"). Podría decirse que los autores llevan a cabo una etnografía muy "a pie de huerto". Según ellos, este movimiento constituye uno de los flancos de un proceso más amplio y en auge de reivindicación del derecho a la ciudad. Realmente, el seguimiento que hacen a las experiencias de este tipo en el área metropolitana de Sevilla, evidencia la condición híbrida y fecunda del llamado "movimiento de los huertos urbanos": porque enlaza luchas patrimoniales por la recuperación de la memoria y la identidad barrial con conocimientos y memoria de inmigrantes campesinos, reivindicaciones de las asociaciones de padres y madres escolares, de las entidades ecologistas, de grupos de consumo basados en la reciprocidad, etc.

Cierra el libro un texto sobre las movilizaciones que conocemos como del 15M. Pero no es otro más de tantos escritos sobre este tema. Tiene un enfoque original, desusado, más allá de la valoración que merezca a quienes lo lean. Nosotros creemos que suscita interrogantes nuevas y muestra ángulos del 15M en los que poco o nada han reparado el tropel de comunicadores y analistas que se han lanzado precipitadamente a escribir sobre el asunto. Como en los demás estudios que reúne el libro, hay etnografía en el estudio de José Luis Moreno sobre las asambleas del 15M. Pero en este caso, la etnografía y los datos, bien traídos, son solo motivo para una reflexión amplia sobre la participación, la democracia y el asambleísmo, válida tanto para este ciclo de movilizaciones como para cualesquiera otros contextos en los que la ciudadanía pretenda implicarse y participar. El autor se remonta a la democracia de los antiguos, para mostrarnos sus rasgos definitorios. Se vale para ello de Aristóteles, pero con apoyo de historiadores del mundo antiguo; contrasta tales rasgos con los de la democracia de los modernos. Y sobre ese juego de contrastes, nos enfrenta a paradojas y antinomias que nos habían pasado desapercibidas y que nos exigen reconsiderar el hecho participativo, no para rechazarlo, sino para hacerlo verdaderamente tal. Por ejemplo: las implicaciones de la cualificación o descualificación política, o del gradiente de unos y otros participantes en esa escala; el tamaño de la asamblea; la voluntariedad o gratuidad como condición de respetabilidad asamblearia y sus efectos excluyentes, distintos, pero tan palmarios como los que 
conlleva la profesionalización; el mundo político extraparlamentario como campo cultural autorreferencial; el chocante ángulo que ilumina la "economía del militante" y las "retribuciones militantes"... En fin, un texto interesante para repensar, como analistas y como ciudadan@s, insospechados cauces de emergencia de aristocracias democráticas. 